\title{
Generating and Mathematical Modelling of Discontinuous Impulse Waveforms
}

\author{
Pavol Štefanec ${ }^{*}$ \\ Faculty of Electrical Engineering \\ University of Žilina \\ Žilina, Slovak Republic \\ Branislav Dobrucký \\ Faculty of Electrical Engineering \\ University of Žilina \\ Žilina, Slovak Republic
}

\author{
Mariana Beňová \\ Faculty of Electrical Engineering \\ University of Žilina \\ Žilina, Slovak Republic
}

\begin{abstract}
Paper deals with mathematical modelling of impulse waveforms and impulse switching functions used in electrical engineering. Impulse rectangular waveforms are created by periodical trigonometric functions with modulo $\pi$, so, the waveforms are discontinuous and strongly non-harmonic ones. Impulse switching functions are investigated using direct- and inverse z-transformation. The results make it possible to present those functions as infinite series expressed in pure numerical-, exponential- or trigonometric forms. Theoretical derived waveforms are compared with simulation worked-out results.
\end{abstract}

Keywords-impulse switching function; Z-transform; inverse Z-transform; modelling and simulation; steady state operation; dynamical state model

\section{MATHEMATiCAl MODELLING OF NON-HARMONIC PERIODICAL DiscontinUOUS FunCTIONS}

It is known that periodical non-harmonic discontinuous function is possible to portray in compact closed form using Fourier infinite series [1]-[2]. It yields for rectangular waveform, Figure 1b,c

$$
\begin{aligned}
& f(t)=\frac{4}{\pi} \sum_{n=0}^{\infty} \frac{1}{2 n+1} \sin [(2 n+1) \omega t] \\
& \text { or } f(t)=(-1)^{2 n+1} \frac{4}{\pi} \sum_{n=0}^{\infty} \frac{1}{2 n+1} \cos [(2 n+1) \omega t]
\end{aligned}
$$

where $\mathrm{t}$ is the time, $\mathrm{n}=0,1,2,3, \ldots$.

$$
\mathrm{F}(s)=\frac{\mathrm{F}_{0}(s)}{s\left(1-\mathrm{e}^{-s \mathrm{~T}}\right)}
$$

where $F_{0}(s)$ is Laplace image of zero period; $F(s)$ - image of total Laplace function $(\mathrm{t} \geq 0)$; $\mathrm{T}$ - time period. Inverse transform defined in complex form as

\author{
Oleg Vyacheslavovich Chernoyarov \\ Department of Radio Engineering Devices, and Antenna \\ Systems \\ Moscow Power Engineering Institute - National Research \\ University \\ Moscow, Russia
}

$$
f(t)=\frac{1}{2 \pi \mathrm{i}} \int_{\mathrm{c}-\mathrm{i} \infty}^{\mathrm{c}+\mathrm{i} \infty} \frac{\mathrm{F}_{0}(s)}{s\left(1-\mathrm{e}^{-s \mathrm{~T}}\right)} \mathrm{d} s
$$

where $\mathrm{i}$ is imaginary unit $\sqrt{-1}$, it is not so easy particularly for higher order systems. Classical solution leads to results in Fourier series form, otherwise the Heaviside calculus is to be used.

One of the lesser known methods is using of FischerTurbar definition of $\operatorname{arc}_{0}$ tan for the main value < $-\frac{\pi}{2} ;+\frac{\pi}{2}>$ based on a standardization of trigonometric function modulo $\pi$ [3]-[4]. So, increasing saw-tooth function with angular frequency $\omega$ can be expressed in closed form

$$
f_{\text {saw }+}(t)=\frac{2}{\pi} \arctan \left[\frac{\sin (\omega t)}{1+\cos (\omega t)}\right]
$$

as can be seen Figure 1a. Similarly, for decreasing saw-tooth waveform

$$
f_{\text {saw }-}(t)=\frac{2}{\pi} \arctan \left[\frac{\sin (\omega t)}{1-\cos (\omega t)}\right] .
$$

Using addition (1) and (2) one obtains a rectangular waveform, Figure $1 \mathrm{~b}$

$$
\begin{aligned}
f_{\text {res }}(t)=\frac{2}{\pi}\left\{\arctan \left[\frac{\sin (\omega t)}{1+\cos (\omega t)}\right]\right. \\
\left.+\arctan \left[\frac{\sin (\omega t)}{1-\cos (\omega t)}\right]\right\} .
\end{aligned}
$$

The saw function (1) converges at $\mathrm{t}=\mathrm{T} / 2$ to 0 value, which is the half of the sum of the left limit and the right one, and it's also valid for rectangular function (3), [6]. Similarly, we can check the convergence of rectangular function at $\mathrm{t}=\pi / 2 \omega$. It yields [7]

$$
f\left(t=\frac{\pi}{2 \omega}\right)=\frac{4}{\pi} \sum_{n=0}^{\infty} \frac{1}{2 n+1} \sin \left[(2 n+1) \omega \frac{\pi}{2 \omega}\right]=1 .
$$




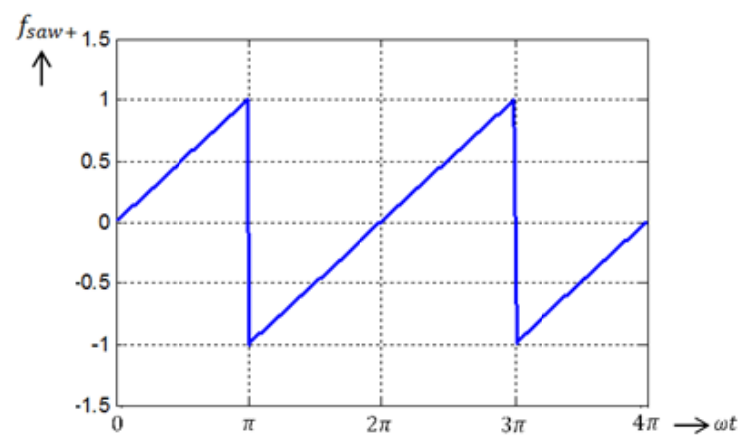

a)

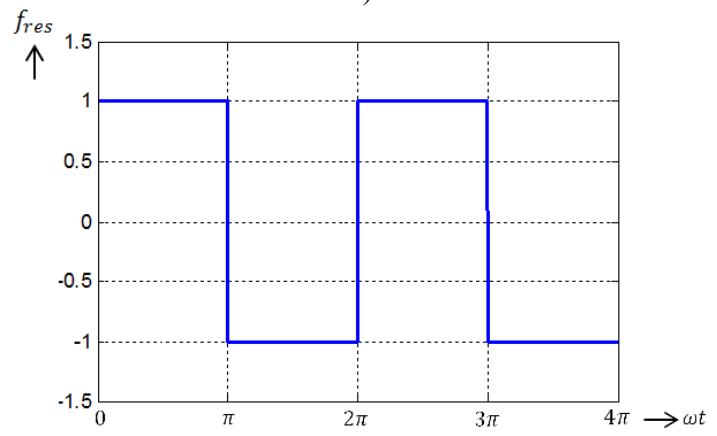

b)

FIGURE I. REPRESENTATION OF SAW-TOOTH WAVEFORM WITH ANGULAR FREQUENCY $\Omega$ (a), AND RECTANGULAR WAVEFORM WITH FULL WIDTH OF ALTERNATING PULSES (b)

\section{CREATING IMPUlSE SWITCHING FUNCTIONS OF NON- HARMONIC DISCONTINUOUS WAVEFORMS}

By changing of pulse width and number of pulses is possible to create various discontinuous waveform using two shifted rectangular waveforms and their subtracting.

Let's shift rectangular function $\mathrm{f}_{\text {res }}(\mathrm{t})$ to the left by $\varphi_{+}$

$$
\begin{aligned}
f_{\varphi+}(t)=\frac{2}{\pi}\{\arctan & {\left[\frac{\sin \left(\omega t+\varphi_{+}\right)}{1+\cos \left(\omega t+\varphi_{+}\right)}\right] } \\
+ & \left.\arctan \left[\frac{\sin \left(\omega t+\varphi_{+}\right)}{1-\cos \left(\omega t+\varphi_{+}\right)}\right]\right\}
\end{aligned}
$$

and shift it to the right by $\varphi_{-}$

$$
\begin{aligned}
f_{\varphi-}(t)=\frac{2}{\pi}\left\{\arctan \left[\frac{\sin \left(\omega t-\varphi_{-}\right)}{1+\cos \left(\omega t-\varphi_{-}\right)}\right]\right. \\
\left.+\arctan \left[\frac{\sin \left(\omega t-\varphi_{-}\right)}{1-\cos \left(\omega t-\varphi_{-}\right)}\right]\right\},
\end{aligned}
$$

whereby $\varphi_{+}$may not be equal to $\varphi_{-}$.

By combination of $\mathrm{f}_{\varphi+}(\mathrm{t})$ and $\mathrm{f}_{\varphi-}(\mathrm{t})$ is possible to create width-controlled waveform or two-pulse-width modulation waveform. For the first one it yields

$$
f_{r e s 1 / 2}(t)=f\left(\frac{f_{\varphi+}+f_{\varphi-}}{2}\right) .
$$

Similarly, it will be created two-pulse waveform using two shifted non-full rectangular functions

$$
f_{2 p_{-} p w m}(t)=f\left(\frac{f_{\varphi_{1}+}+f_{\varphi_{1}-}}{2}+\frac{f_{\varphi_{2}+}+f_{\varphi_{2}-}}{2}\right)
$$

Let's create - at first - non-full rectangular waveform with impulse width $\pi / 2$ i.e. $\varphi_{-}=0 ; \varphi_{+}=\pi / 2$ :

$$
\begin{array}{r}
f_{\varphi+}(t)=\frac{2}{\pi}\left\{\arctan \left[\frac{\sin (\omega t+\pi / 2)}{1+\cos (\omega t+\pi / 2)}\right]\right. \\
\left.+\arctan \left[\frac{\sin (\omega t+\pi / 2)}{1-\cos (\omega t+\pi / 2)}\right]\right\} \\
f_{\varphi-}(t)=\frac{2}{\pi}\left\{\arctan \left[\frac{\sin (\omega t-0 \pi)}{1+\cos (\omega t-0 \pi)}\right]\right. \\
\left.+\arctan \left[\frac{\sin (\omega t-0 \pi)}{1-\cos (\omega t-0 \pi)}\right]\right\}, \\
f_{\text {res } 1 / 2}(t)=f\left(\frac{f_{\pi / 2}+f_{0 \pi}}{2}\right), \text { Figure } 2 \mathrm{a} .
\end{array}
$$

The second two-pulse waveform needs to create two such non-rectangular waveforms:

$$
\begin{aligned}
& f_{\varphi_{1}+}(t)=\frac{2}{\pi}\left\{\arctan \left[\frac{\sin \left(\omega t+\frac{3 \pi}{4}\right)}{1+\cos \left(\omega t+\frac{3 \pi}{4}\right)}\right]\right.\left.+\arctan \left[\frac{\sin (\omega t+3 \pi / 4)}{1-\cos (\omega t+3 \pi / 4)}\right]\right\} \\
& f_{\varphi_{1}-}(t)= \frac{2}{\pi}\left\{\arctan \left[\frac{\sin (\omega t-0 \pi)}{1+\cos (\omega t-0 \pi)}\right]\right. \\
&\left.\quad+\arctan \left[\frac{\sin (\omega t-0 \pi)}{1-\cos (\omega t-0 \pi)}\right]\right\} \\
& f_{\varphi_{2}+}(t)=\frac{2}{\pi}\left\{\arctan \left[\frac{\sin (\omega t+\pi / 4)}{1+\cos (\omega t+\pi / 4)}\right]\right. \\
&\left.\quad+\arctan \left[\frac{\sin (\omega t+\pi / 4)}{1-\cos (\omega t+\pi / 4)}\right]\right\} \\
& f_{\varphi_{2}-}(t)=\frac{2}{\pi}\left\{\arctan \left[\frac{\sin (\omega t-\pi / 2)}{1+\cos (\omega t-\pi / 2)}\right]\right. \\
&\left.\quad+\arctan \left[\frac{\sin (\omega t-\pi / 2)}{1-\cos (\omega t-\pi / 2)}\right]\right\} \\
& f_{2 p_{-} p w m}(t)=f\left(\frac{\left.f_{\varphi_{1}+}+f_{\varphi_{1}-}+\frac{f_{\varphi_{2}+}+f_{\varphi_{2}-}}{2}\right), \text { Figure } 2 \mathrm{~b} .}{2}\right.
\end{aligned}
$$




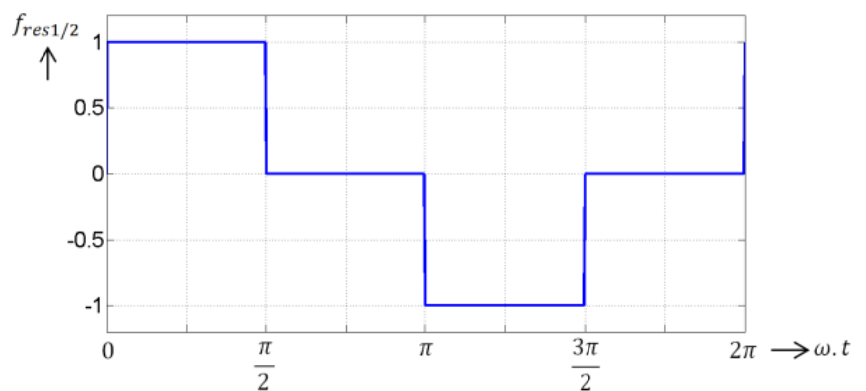

a)

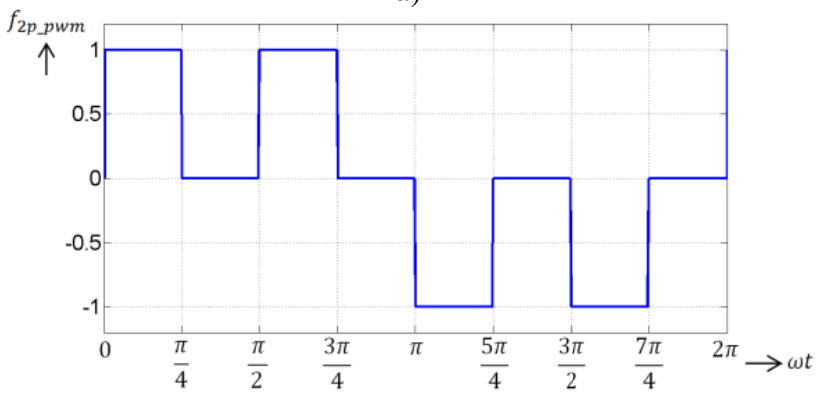

b)

FIGURE II. RECTANGULAR WAVEFORM WITH HALF WIDTH OF ALTERNATING PULSES (a) AND TWO-PULSE MODULATION WAVEFORM (b)

Assuming finite switch-on and switch-off times of realtime waveforms and using normalized derivative pulses we can create impulse switching function for given waveforms, Figure 3.a,b.

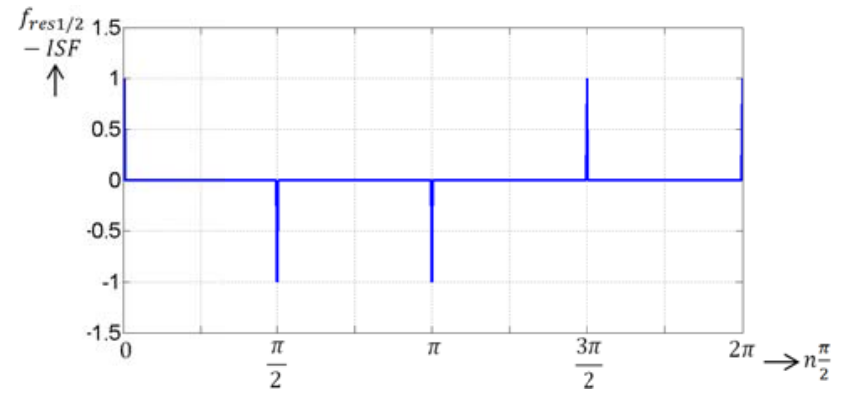

a)

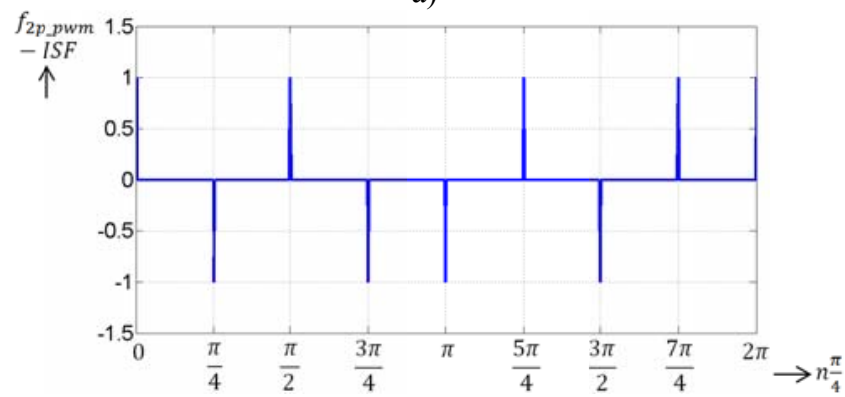

b)

FIGURE III. RECTANGULAR WAVEFORM WITH HALF WIDTH OF ALTERNATING PULSES (a) AND TWO-PULSE MODULATION WAVEFORM (b)

Further, based on zero order hold function [8] and unipolar modulation [12] the switch-off impulses will be substitute by zero points, and result waveforms can be presented as following, Figure 4a,b.

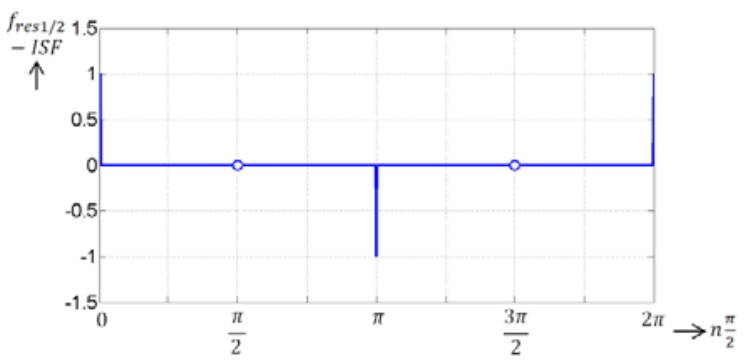

a)

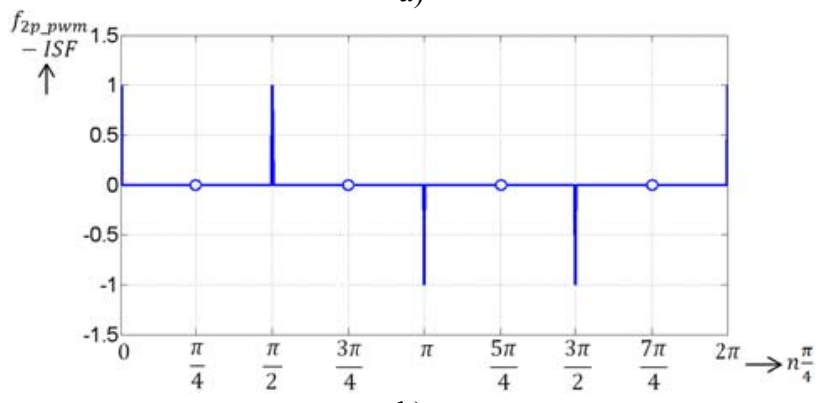

b)

FIGURE IV. IMPULSE SWITCHING FUNCTIONS WITH UNIPOLAR CONTROL OF: RECTANGULAR WAVEFORM WITH HALF WIDTH (a), TWO-PULSE MODULATION WAVEFORM (b)

III. DESCRIPTION OF IMPULSE SWITCHING FUNCTIONS OF NON-HARMONIC DISCONTINUOUS WAVEFORMS IN Z-DOMAIN

Using basic definition of Z-transform - taking into account $Z$-images of constant and alternating series - and based on the rules of the Z-transform it can be write [8]

$$
F(z)=\sum_{n=0}^{+\infty}(-1)^{n} z^{-n}=\sum_{n=0}^{+\infty}(-z)^{-n}
$$

The sum of that geometric series with quotient $\mathrm{z}^{-1}$ is

$$
F(z)=\frac{1}{1+z^{-1}}=\frac{z}{z+1} ; \quad \rightarrow \quad F_{\text {rect }}(z)=\frac{z}{z+1}
$$

where root of the denominator is $\mathrm{z}=-1$.

For inverse Z-transform $\mathrm{F}(\mathrm{z}) \leftrightarrow\left\{\mathrm{f}_{\mathrm{n}}\right\}$ one can use [9], [10]:

- convolution or decomposition of $\mathrm{F}(\mathrm{z})$ on partial fractions (for simpler cases),

- derivatives of $\mathrm{F}(\mathrm{z})$ function

$$
\left\{f_{n}\right\}=\frac{1}{n !} \frac{\mathrm{d}^{n}}{\mathrm{~d} z^{n}} F\left(\frac{1}{z}\right)_{z=0},
$$

- algebraic dividing of polynomials by polynomials

$$
F(z)=\frac{A(z)}{B(z)}=\frac{a_{0} z^{0}+\cdots}{b_{0} z^{0}+\cdots}
$$


- Cauchy integral residua theorem

$$
\left\{f_{n}\right\}=\frac{1}{2 \pi \mathrm{i}} \oint F(z) z^{n-1}=\sum_{k=1}^{N} \underset{z=z_{k}}{\operatorname{res}} F_{r e s}(z) z^{n-1}
$$

Using residua theorem

$$
\begin{aligned}
\sum_{k=1}^{N} \underset{z=z_{k}}{\operatorname{res}} F_{r e s}(z) z^{n-1} & =\sum_{k=1}^{N} \lim _{z \rightarrow z_{k}}\left(z-z_{k}\right) F_{r e s}(z) z^{n-1}= \\
& =\sum_{k=1}^{N} \frac{A\left(z_{k}\right)}{B^{\prime}\left(z_{k}\right)} z_{k}^{n-1},
\end{aligned}
$$

where $\mathrm{k} \in\langle 1, \ldots \mathrm{N}\rangle ; \mathrm{n}=0,1,2, \ldots \infty ; \mathrm{N}$ is number of poles of denominator and $\mathrm{B}^{\prime}$ is derivative of denominator

$$
\frac{\mathrm{d} B(z)}{\mathrm{d} z}\left(\text { at } z=z_{k}\right)
$$

Then

$$
f_{\text {res }}(n) \equiv\left\{f_{n}\right\}_{\text {res }}=(-1)^{n}=e^{j n \pi}=\cos (n \pi) .
$$
[8]

Similarly, based on $1 / 2$-pulse length and following relation

$$
F(z)=\frac{z^{N}}{z^{N}+1}
$$

So, for $1 / 2$-pulse length and using above relation the $z$ image of the half-rectangular waveform will be:

$$
F_{\text {res } 1 / 2}(z)=\frac{z^{2}}{z^{2}+1}
$$

where roots of the denominator are $\mathrm{z}_{1,2}= \pm \mathrm{i}$ are placed on boundary of stability in unit circle [1], [9], Figure 5a.

Applying inverse z-transform we can write

$$
\begin{aligned}
f_{\frac{r e s 1}{2}(n)} & =\sum_{k=1}^{2} \frac{A\left(z_{k}\right)}{B^{\prime\left(z_{k}\right)}} z_{k}^{n-1}=\sum_{k=1}^{2} \frac{z_{k}}{2 z_{k}} z_{k}^{n}= \\
= & \frac{1}{2}\left[\left(z_{1}\right)^{n}+\left(z_{2}\right)^{n}\right]=\frac{1}{2} \mathrm{i}^{n}\left[1+(-1)^{n}\right]
\end{aligned}
$$

This result can be expressed in different forms: pure numerical, exponential, and trigonometric ones

$$
\begin{aligned}
f_{\text {res } 1 / 2}(n) & =\frac{1}{2}\left\{e^{\mathrm{i} n \frac{\pi}{2}}+e^{-\mathrm{i} n \frac{\pi}{2}}\right\} \\
& =1 \cos \left(n \frac{\pi}{2}\right)
\end{aligned}
$$

Similarly for two-pulse modulation waveform

$$
F_{2 p \_p w m}(z)=\left(\frac{z^{4}}{z^{4}+1}+z^{-2} \frac{z^{4}}{z^{4}+1}\right)=\frac{z^{4}+z^{2}}{z^{4}+1}
$$

where roots of polynomial of the denominator are

$$
z_{1,2}= \pm e^{ \pm \mathrm{i} \frac{\pi}{4}}, \text { so } z_{1,2}=e^{ \pm \mathrm{i} \frac{\pi}{4}} ; \quad z_{3,4}=-e^{ \pm \mathrm{i} \frac{\pi}{4}}
$$

placed again on boundary of stability in unit circle Figure $5 \mathrm{~b}$.

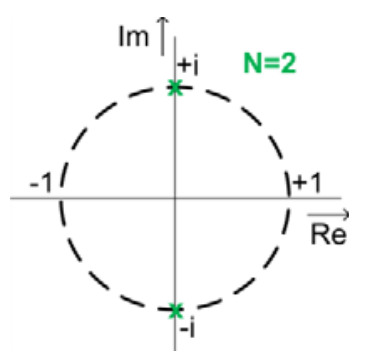

a)

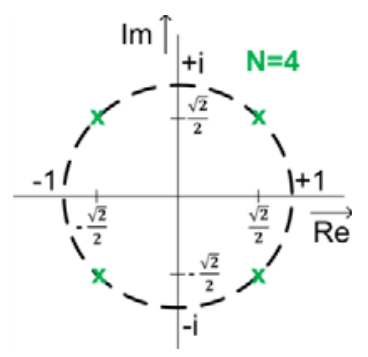

b)
FIGURE V. POLE PLACEMENTS OF DENOMINATOR POLYNOMIALS OF F_(RES1/2) (Z) - (a) AND F_(2P_PWM) (Z) -

Applying inverse Z-transform for converter output phase voltages in $Z$-domain one can create impulse switching functions. For inverse Z-transform $F(\mathrm{z}) \leftrightarrow\{f\}$ one can use the residua theorem described above.

Then

$$
\begin{aligned}
& f_{2 p_{p w m}}(n)=\sum_{k=1}^{4} \frac{A\left(z_{k}\right)}{B^{\prime\left(z_{k}\right)}} z_{k}^{n-1}=\sum_{k=1}^{4} \frac{z_{k}^{3}+z_{k}^{1}}{4 z_{k}^{3}} z_{k}^{n} \\
&=\frac{1}{4} \sum_{k=1}^{4}\left(1+z_{k}^{-2}\right) z_{k}^{n}= \\
&=\frac{1}{4}\left\{\left[e^{\mathrm{i} n \frac{\pi}{4}}+e^{-\mathrm{i} n \frac{\pi}{4}}+(-1)^{n} e^{\mathrm{i} n \frac{\pi}{4}}+(-1)^{n} e^{-\mathrm{i} n \frac{\pi}{4}}\right]\right. \\
&+\left[-\mathrm{i} e^{\mathrm{i} n \frac{\pi}{4}}+\mathrm{i} e^{-\mathrm{i} n \frac{\pi}{4}}-\mathrm{i}(-1)^{n} e^{\mathrm{i} n \frac{\pi}{4}}\right. \\
&\left.\left.+\mathrm{i}(-1)^{n} e^{-\mathrm{i} n \frac{\pi}{4}}\right]\right\}
\end{aligned}
$$

$$
=\frac{1}{2}\left[1+(-1)^{n}\right] \cos \left(n \frac{\pi}{4}\right)+\frac{1}{2}\left[1+(-1)^{n}\right] \sin \left(n \frac{\pi}{4}\right) \text {. }
$$

After adapting

$$
f_{2 \mathrm{p} \_ \text {pwm }}(n)=\frac{1}{2}\left[1+(-1)^{n}\right] \sqrt{2} \sin \left(n \frac{\pi}{4}+\frac{\pi}{4}\right) .
$$

Graphical results using inverse z-transform are presented in Figure 6a,b. 


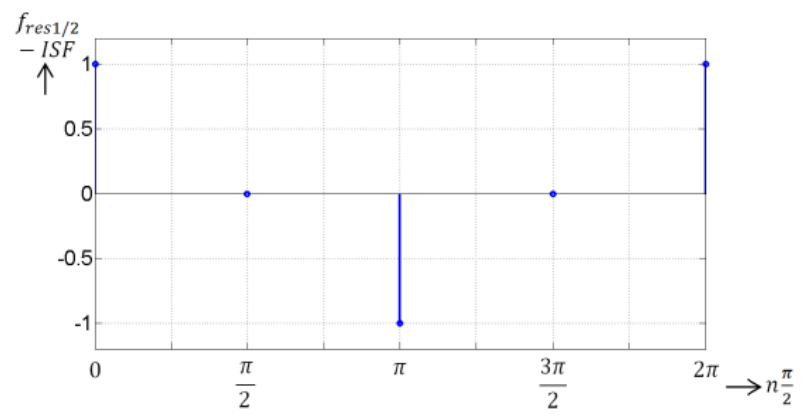

a)

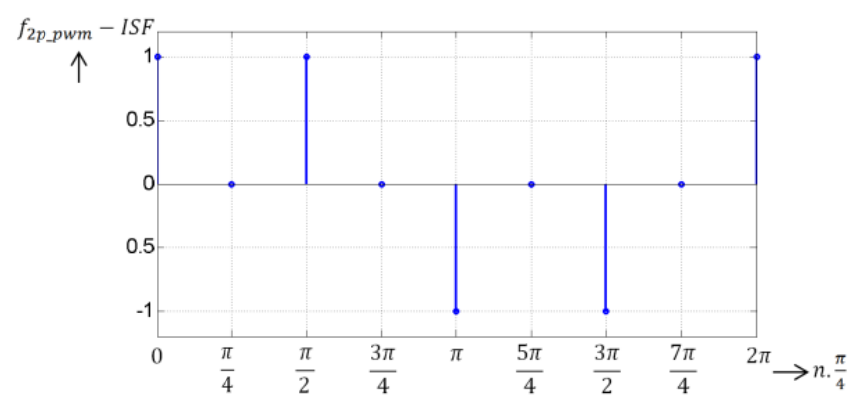

b)

FIGURE VI. IMPULSE SWITCHING FUNCTION WORKEDOUT USING $f_{\text {res } 1 / 2}\left(n \frac{\pi}{2}\right)$ a), AND $f_{2 \text { p_pwm }}\left(n \frac{\pi}{4}\right)$ b)

\section{GENERATING AND MODELling OF NON-HARMONIC} PERIODICAL EXCITING FUNCTIONS BASED ON ISF

Dynamical state model of the systems include exciting functions $\mathrm{u}(\mathrm{t})$ as input vector. The models can be expressed in a continuous or discrete form:

$$
\begin{gathered}
\frac{\mathrm{d}}{\mathrm{d} t} x=\boldsymbol{A} x(t)+\boldsymbol{B} u(t) \\
\text { or } \\
x_{\mathrm{k}+1}=\boldsymbol{F} x_{\mathrm{k}}+\boldsymbol{G} u_{\mathrm{k}}
\end{gathered}
$$

respectively, where $\mathrm{k}$ is order of computation step (not step of sequence).

Discrete form of state space model of the investigated system with the step of impulse switching function can be obtained directly from the impulse switching functions generated and above:

$$
\boldsymbol{x}(n . \text { step })=\boldsymbol{A} x(t)+\boldsymbol{B} u(n . \text { step }), \quad n=0,1,2, \ldots \infty
$$

where the step is equal to the step or period, respectively of the impulse sequences $T_{p}$ of switching functions

$$
\left\{u_{n}\right\} \equiv u_{n}(n . s t e p)=u_{n}\left(n \cdot T_{p}\right) \text {. }
$$

It being understood that sequence period $\mathrm{T}_{\mathrm{p}}$ may not be consistent with the time period of the waveform $\mathrm{T}$ (preferably, if it is less).

To generate a continuous form of exciting functions $\mathrm{u}_{\mathrm{k}} \equiv \mathrm{u}(\mathrm{k} \Delta)$ was created following procedure and there were used already created impulse switching function $\left\{\mathrm{f}_{\mathrm{n}}\right\} \equiv \mathrm{f}(\mathrm{n})$ :

$$
\begin{gathered}
f_{\text {res } 1 / 2}(n)=1 \cos \left(n \frac{\pi}{2}\right) ; \\
f_{2 p_{-} p w m}(n)=\frac{1}{2}\left[1+(-1)^{n}\right] \sqrt{2} \sin \left(n \frac{\pi}{4}+\frac{\pi}{4}\right) .
\end{gathered}
$$

Exciting functions $\mathrm{u}_{\mathrm{k}} \equiv \mathrm{u}(\mathrm{k} \Delta)$ was created based on zero order hold function [8] and the approach is as following:

- Index of the sequence $n$ will be replaced by an integer presentation of the ratio of continuous variable $\omega t$ and step or sequence period $T_{\mathrm{p}}$, respectively -

$(\pi / 2$ or $\pi / 2)$ :

$$
n=\operatorname{integer}\left(\frac{\omega t}{\text { step }[\mathrm{rad}]}\right) .
$$

- Creation of the share will be converted into a timemeasure by substitution $\pi \rightarrow \omega T / 2$

$$
n=\operatorname{integer}\left(\frac{\omega t}{\left.s t e p\right|_{\pi \rightarrow \omega T / 2}[\mathrm{~s}]}\right),
$$

- continuous time variable $\omega t$ will be replaced by discretized one: $t \approx k . \Delta$

where $\Delta$ is a step in the calculation of the digital computer and the $\mathrm{k}$ is the order of calculation steps. Thus

$$
n=\operatorname{integer}\left(\frac{t}{\operatorname{step}[\mathrm{s}]}\right) \approx \operatorname{integer}\left(\frac{\Delta}{\operatorname{step}[\mathrm{s}]} k\right) \text {. }
$$

Examples of creation of exciting function $\boldsymbol{u}_{k} \equiv \boldsymbol{u}(k \Delta)$ :

Let's take into account mentioned discretized impulse switching functions (sequence)

$$
f_{\operatorname{res} 1 / 2}(n)=1 \cos \left(n \frac{\pi}{2}\right) \text { with step }=\frac{\pi}{2}
$$

and

$$
\begin{gathered}
f_{2 \mathrm{p}_{\mathrm{p}} \mathrm{pwm}}(n)=\frac{1}{2}\left[1+(-1)^{\mathrm{n}}\right] \sqrt{2} \sin \left(n \frac{\pi}{4}+\frac{\pi}{4}\right) \text { with step } \\
=\frac{\pi}{4} .
\end{gathered}
$$

Then continuous excitation function $\mathrm{f}_{\mathrm{res} 1 / 2}(\mathrm{t})$ will be

$$
\begin{aligned}
& f_{\text {res } 1 / 2}(t)=\cos [\text { integer } \left.\left(\frac{\omega t}{\pi / 2}\right) \frac{\pi}{2}\right]=\cos \left[\operatorname{integer}\left(\frac{4 t}{T}\right) \frac{\pi}{2}\right] \\
&=\cos \left[\operatorname{integer}(4 f t) \frac{\pi}{2}\right] .
\end{aligned}
$$

After time discretization with step of $\Delta$ when $\mathrm{t}=\mathrm{k} . \Delta$

$$
f_{\text {res } 1 / 2}(k)=\cos \left[\operatorname{integer}\left(4 \frac{\Delta}{T} k\right) \frac{\pi}{2}\right] \text {. }
$$

In case of further function $\mathrm{f}_{2 p_{\text {p }} \text { pwm }}(\mathrm{t})$

$f_{2 \mathrm{p} \_ \text {pwm }}(n, t)=\frac{1}{2}\left[1+(-1)^{n}\right] \sqrt{2} \sin \left[\operatorname{integer}(8 f t) \frac{\pi}{4}+\frac{\pi}{4}\right]$.

Or 


$$
\begin{aligned}
f_{2 \mathrm{p}_{\mathrm{pwm}}} & (n, k)= \\
= & \frac{1}{2}\left[1+(-1)^{n}\right] \sqrt{2} \sin \left[\text { integer }\left(8 \frac{\Delta}{T} k\right) \frac{\pi}{4}+\frac{\pi}{4}\right]
\end{aligned}
$$

for any $n$ and $k \in<0$; step $/ \Delta>$.

Another approach is using of substitution

$$
(-1)^{n}=(-1)^{\text {integer }\left(8 \Delta_{T} k\right)}
$$

Then we obtain function dependency on $k$

$$
\begin{gathered}
f_{2 \mathrm{p}_{\mathrm{pwm}}}(k)=\frac{1}{2}\left[1+(-1)^{\mathrm{integer}\left(8 \frac{\Delta}{T} k\right)}\right] \\
. \sqrt{2} \sin \left[\text { integer }\left(8 \frac{\Delta}{T} k\right) \frac{\pi}{4}+\frac{\pi}{4}\right] .
\end{gathered}
$$

Now, we can draw the function for $\mathrm{k} \in<0 ; \infty>$ thus it can be solved dynamical state of the system given by Eqs. (4), (5), [11]-[12] continuously with the $\Delta$ computation step, Figure $7 \mathrm{a}, \mathrm{b}$.

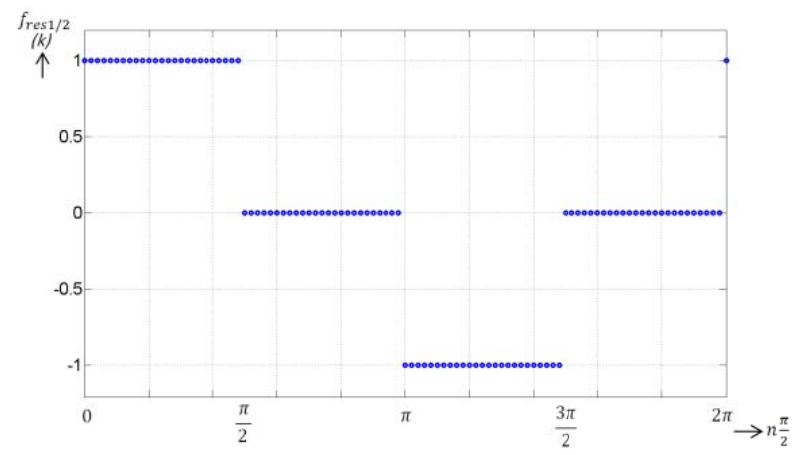

a)

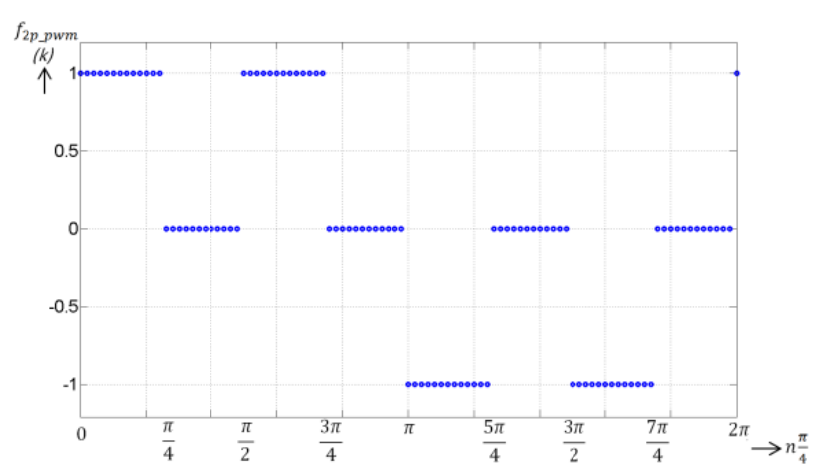

b)

FIGURE VII. FUNCTIONS $f_{\text {RES } 1 / 2}(k)$ (a), AND $f_{2 \text { P_PWM }}(k)$ (b)

Note: Under the same computation step the waveforms in Figure 7 are the same as those continuous ones in Figure 2 what is the approving of above equations (6), (7).

\section{CONCLUSION}

Using periodical properties of the function $\operatorname{arc}_{0} \tan$ modulo $\pi$ we can generate various (any) rectangular impulse functions with any width and numbers of impulses. Taking in account of finite rising slope of the impulses one can generate the impulse switching functions in unipolar or bipolar mode for utilization in electrical systems. Application of unipolar modulation and zero order function it is possible to obtain functions which can be describable by ztransformation. Then, using inverse $z$-transform we obtain unipolar switching impulse functions as dependency on order of the step of sequence in z-plain. Besides, by substituting step of sequence by integer variable function, it is possible to obtain solution in continuous form. Thus, unlikely to pure numerical computing, ISFs make it possible to calculate variable quantities at any time instants. Presented techniques are suitable for both transient and steady-state behavior of investigated system mainly in electrical engineering.

\section{ACKNOWLEDGMENT}

The paper was supported from Slovak Grant Agency VEGA by the grant No. 1/0928/15 and the Russian Science Foundation by the grant No. 14-49-00079.

\section{REFERENCES}

[1] J. G. Aramovich, G. L. Lunts, L. C. Elsgolts: Functions of Complex Variable, Operator Calculus, Stability Theory (in Russian - translated into Slovak), Alfa, Bratislava, 1973.

[2] R.J. Beerends, H.G. Morsche, J.C. Berg, E.M. Vrie: Fourier and Laplace Transforms. Publisher: Cambridge University Press, 2003.

[3] T.J. Takeuchi: Method of $\Phi$-Function to Analyses of SCR Rectifier Circuit. In: Journal of IEEJ, Japan, Vol. 83-10, No. 901, 1963.

[4] K.A. Turbar: Representation discontinuous periodical currents and voltages under their developing into the series (in German). ETZ-A journal, Vol. 93 (1972), No. 2, pp. 57-60.

[5] J. Fischer: Lectures on $\operatorname{arc}_{0} \tan$ for the main value based on a standardization of trigonometric functions (in German). University of Karlsruhe (KIT), 1972.

[6] J. Škrášek, Z. Tichý: Basics of applied mathematics II (in Czech). SNTL Publisher, Praha, 1986.

[7] B. Dobrucký, O.V. Chernoyarov, M. Marčoková: Computation of the total harmonic distortion of impulse system quantities using infinite series. Proceedings of 14th Conference on Applied Mathematics, STU Bratislava, Feb 2015, pp. 213 - 220.

[8] R. Vích: Z-transformation and some of its Utilization (in Czech) SNTL Mathematical Seminar, Prague, 1983.

[9] I.V. Blagouchine, E. Moreau: Analytic method for the computation of the total harmonic distortion by the Cauchy method of residues. IEEE Transactions on Communications, Vol. 59, No. 9, Sept. 2011: s. 24782491.

[10] J. Moravčík: Mathematical Analysis 3 (in Slovak), Textbook, Alfa Publishing House, Bratislava, 1992, ISBN 80-05-01044-3.

[11] B. Dobrucký, M. Pokorný, M. Beňová: On Impulse Switching Functions of Inverters as an Orthogonal System. Journal of Mathematics and System Science (USA), ISSN 2159-5291, Vol. 4 (2014), No. 12, pp. 743-749.

[12] B. Dobrucký, M. Pokorný, M. Beňová, M. A. R. Abdamula: Modeling of power converters using Z-transform. Communication - Scientific Letters of the University of Zilina, 2013, vol. 15, no. 3, pp. 43-47. 